\title{
Assessment of RSS Model Calibration with Real WLAN Devices
}

\author{
J. Manuel Castro-Arvizu ${ }^{\ddagger}$, Ana Moragrega ${ }^{\dagger}$, Pau Closas $^{\dagger}$, Juan A. Fernández-Rubio ${ }^{\ddagger}$ \\ $\ddagger$ Universitat Politècnica de Catalunya (UPC) Campus Nord, 08034 Barcelona (Spain) \\ ${ }^{\dagger}$ Centre Tecnològic de Telecomunicacions de Catalunya (CTTC) Av. Carl Friedrich Gauss 7, 08860 \\ Castelldefels, Barcelona (Spain)
}

\begin{abstract}
Received Signal Strength (RSS) for indoor localization is widely used due to its simplicity and availability in most mobile devices. The RSS channel model is defined by the propagation losses and the shadow fading. This paper studies twoslope RSS channel model and compares its validity to classical one-slope path loss model. Particularly, the work presents realdata results of a Bayesian calibration method. Validation of the model fitting is then performed in a dynamic scenario where the distance to a reference node is tracked by a Kalman Filter. Results show the superiority of two-slope model, specially at large distances.
\end{abstract}

Keywords-Indoor localization, robust filtering, two-slope path loss model, bayesian inference, extended kalman filter, model calibration, received signal strength

\section{INTRODUCTION}

Navigation and location technologies have been reaching in a major interest where Global Navigation Satellite System (GNSS) is mostly adopted. The limitation with this kind of technology is his principal feature: direct "sky view". Because of this, GNSS cannot satisfy the high accuracy positioning requirements for many applications in engineering and mining surveying, structural monitoring, urban and indoor positioning. However, the growth in indoor applications has focused the research in new techniques for attempting mitigate the poor GNSS performance on this type of environments.

Nowadays, a multitude of emerging technologies are based on signals of opportunity as Wireless Local Area Network (WLAN), Ultra Wide-Band (UWB), Zigbee, and other used for indoor localization and tracking. In terms of cost and ability, Wireless-based indoor location is widely used due to the already deployment of Anchor Points (AP) in urban and indoor areas. This work is focused on IEEE.802.11x signals as principal approach for the localization problem.

There are several methods for indoor positioning purposes e.g ToA (Time of Arrival), RSS measurements, AoA (Angle

This work has been partially supported by the Spanish Ministry of Economy and Competitiveness project TEC2012-39143 (SOSRAD), by the European Commission in the Network of Excellence in Wireless COMmunications NEWCOM $\sharp$ (contract n. 318306), and by Generalitat de Catalunya under grant 2014-SGR-1567. This work is supported by the "Ministerio de Economía y Competitividad" of the Spanish Government and ERDF funds (TEC201341315-R DISNET), and the Catalan Government (2014 SGR 60 AGAUR) and the National Council of Science and Technology (CONACyT) of the Mexican Government of Arrival) and so on. This work is centered in WLAN RSSbased positioning systems. The first step for indoor location is the distance estimation between the user and the AP. Several indoor positioning techniques are found in literature for this task [1]-[3].

Most of the network-based location estimations use RSS measurements because almost all mobile devices are afforded to use this type of measurements. Theoretical and empirical models are used to translate the difference between the transmitted and Received Signal Strength into an estimated range [1]-[5].

Although fingerprinting techniques are widely used [6]-[8] also, this paper is focused only on geometric or statistical techniques that are based on previous knowledge of the radio propagation channel model.

A Propagation model could built the radio map and also report changes in the environment. There are several models in the literature to characterize this channel [9], [10]. This work considers the IEEE $802.11 x$ channel model.

Indoor RSS-based localization has become a popular solution, but standard techniques still consider a time invariant signal model with a priori known constant parameters. This standard RSS-based localization problem with known APs positions, a simple single slope path loss model and known model parameters, has already been addressed in the literature using standard/fusion solutions [1], [4], [11]. While some contributions considered the RSS-based localization problem using a single path loss model with unknown parameters [5], [12][15], the general solution that considers a generalized distance dependent measurement model is an important missing point.

Usually, indoor location systems operate under Non-LineOf-Sight (NLOS) scenarios. These conditions could cause an error in the distance estimation and accordingly a bad location accuracy. Approaches to avoid this problem was conducted for UWB signals. In literature, some NLOS mitigation techniques have obtained significant results in distance estimation using real signal but only considering the classical one-slope model [16]-[18].

In this work, a two-slope path loss model [19] is considered and validates the channel calibration algorithm with an online distance estimation proposed in [20]. Once the RSS model is calibrated, an Extended Kalman Filter (EKF) is used for distance estimation with real RSS measurements in a typical office environment, highlighting the validity of our approach. 
The remainder of the paper is organized as follows. The mathematical formulation of the system is given in Section II, the proposed technical solution is detailed in Section III and IV. Illustrative results are discussed in Section VI and Section VII concludes the paper with final remarks.

\section{Channel MODELING}

The widely used model for RSS observations is the path loss model, which is a simple yet realistic model for such measurements. Path loss is the reduction in signal strength over distance. The path loss depends specifically on the distance between the transmitter (Anchor Point) and the receiver (Mobile Target) [21]. Indeed, it has been observed that for far distances ( $5 \leq d \leq 30$ meters), there is a steeper overall drop in the RSS at the receiver. This effect is due to path reflections from the environment. For this particularity, an extension of the classical path loss model accounting for two regions of propagation, referred to as the two-slope model [19] is considered.

Under this model, the RSS in the mobile target is modeled as [19]:

$$
\operatorname{RSS}(d) \triangleq y(d)= \begin{cases}h^{(1)}(d)+\chi_{\sigma_{1}^{2}} & \text { if } d \leq d_{b p} \\ h^{(2)}(d)+\chi_{\sigma_{2}^{2}} & \text { if } d>d_{b p}\end{cases}
$$

where $d$ is the relative distance between the Anchor Point (AP) and the Mobile Target, and

$$
\begin{aligned}
& h^{(1)}(d)=L_{0}+10 \alpha_{1} \log _{10}(d) \\
& h^{(2)}(d)=L_{0}+10 \alpha_{1} \log _{10}\left(d_{b p}\right)+10 \alpha_{2} \log _{10}\left(d / d_{b p}\right) .
\end{aligned}
$$

where, $L_{0}$ is the RSS in a reference distance [19], [20] The first equation gives the path loss (expressed in $\mathrm{dB}$ ) for close distances ( $d \leq d_{b p}$, known as the breakpoint distance) and the second equation gives the path loss beyond $d_{b p}$. The $\alpha_{1}$ and $\alpha_{2}$ values are referred to as path loss exponents, defining the slopes before and after $d_{b p}$, respectively.

Depending on the transmitter/receiver geometrical configuration, the RSS values might be distorted for the nominal. This variation (known as shadow fading) is modeled as $\chi_{\sigma_{i}^{2}} \sim \mathcal{N}\left(0, \sigma_{i}^{2}\right)$. Similarly as the path loss exponents, the variance differ before and after the breakpoint distance. To sum up, the two-slope RSS measurement model is parameterized and fully determined by $\boldsymbol{\psi}=\left[\alpha_{1}, \alpha_{2}, \sigma_{1}^{2}, \sigma_{2}^{2}, d_{b p}\right]^{\top}$.

\section{MOdEl PARAMETER ESTIMATION By BAYESIAN INFERENCE}

This section summarizes the estimation method for model parameters introduced in [20]. The statistical problem is to detect the change point in the means and variances of the RSS measurements, as well as estimating the other model parameters. The measurements follow the Gaussian distribution discussed in Section II. In this paper a distribution that can be factorized as:

$$
\boldsymbol{\psi} \sim \pi(\boldsymbol{\psi})=\pi\left(\alpha_{1}\right) \pi\left(\alpha_{2}\right) \pi\left(\sigma_{1}^{2}\right) \pi\left(\sigma_{2}^{2}\right) \pi\left(d_{b p}\right)
$$

is asumed.
An uniform prior over the full range of possible distances (defined as $d_{\max }$ ) is considered for the $d_{b p}$, and conjugated priors are given to the path losses and the variances:

$$
\begin{aligned}
& \alpha_{i} \sim \pi\left(\alpha_{i}\right)=\mathcal{N}\left(0, \mathcal{V}_{\alpha_{i}}^{2}\right) \\
& \sigma_{i}^{2} \sim \pi\left(\sigma_{i}^{2}\right)=\Gamma^{-1}\left(a_{i}, b_{i}\right) \\
& d_{b p} \sim \pi\left(d_{b p}\right)=\mathcal{U}\left(0, d_{\max }\right),
\end{aligned}
$$

$\mathcal{V}_{\alpha_{i}}^{2}, a_{i}$ and $b_{i}$ control the initial uncertainty on the parameters of the model. Since little knowledge is assumed, $\mathcal{V}_{\alpha_{i}}^{2}=0.0001$, $a_{i}=0.1$ and $b_{i}=0.0001$ values are used in the results section.

The problem of inferring the posterior distribution over the latent variables $\alpha_{1}, \sigma_{1}^{2}, \alpha_{2}$ and $\sigma_{2}^{2}$ could be solved analytically via Bayes theorem [22]. However, due to the unknown $d_{b p}$, computational methods such as Markov Chain Monte Carlo (MCMC) methods are needed. The MCMC algorithm combines the prior distribution with the likelihood to obtain the posterior distribution. MCMC algorithms are typically run for a large number of iterations (to achieve the convergence to the target posterior) [20], [23].

The statistical model was implemented with Gibbs Sampling [24], which provides the joint posterior distribution of interest. To be implemented, the Gibbs sampler requires the posterior conditional for each of the latent variables. They are obtained as [20]

$$
\begin{aligned}
& \alpha_{1} \sim p\left(\alpha_{1} \mid \sigma_{1}^{2}, \alpha_{2}, \sigma_{2}^{2}, d_{b p}, \boldsymbol{y}\right)=\mathcal{N}\left(\alpha_{1, o}, \sigma_{1,0}^{2}\right), \\
& \sigma_{1}^{2} \sim p\left(\sigma_{1}^{2} \mid \alpha_{1}, \alpha_{2}, \sigma_{2}^{2}, d_{b p}, \boldsymbol{y}\right)=\Gamma^{-1}\left(a_{1, o}, b_{1, o}\right), \\
& \alpha_{2} \sim p\left(\alpha_{1} \mid \sigma_{1}^{2}, \alpha_{2}, \sigma_{2}^{2}, d_{b p}, \boldsymbol{y}\right)=\mathcal{N}\left(\alpha_{2 . o}, \sigma_{2,0}^{2}\right), \\
& \sigma_{2}^{2} \sim p\left(\sigma_{1}^{2} \mid \alpha_{1}, \alpha_{2}, \sigma_{2}^{2}, d_{b p}, \boldsymbol{y}\right)=\Gamma^{-1}\left(a_{2, o}, b_{2, o}\right), \\
& d_{b p} \sim p\left(d_{b p} \mid \alpha_{1}, \sigma_{1}^{2}, \alpha_{2}, \sigma_{2}^{2}, \boldsymbol{y}\right) \propto p(\boldsymbol{y} \mid \psi) \cdot \pi\left(d_{b p}\right),
\end{aligned}
$$

where,

$$
\begin{gathered}
\left.\alpha_{1, o}=20 \frac{\sigma_{1,0}^{2}}{\sigma_{1}^{2}}\left(\sum_{n=1}^{N_{d b p}} y_{n} \log _{10} d_{n}-L_{o} \sum_{n=1}^{N_{d b p}} \log _{10} d_{n}\right)\right) \\
\sigma_{1,0}^{2}=\frac{\sigma_{1}^{2} \mathcal{V}_{\alpha_{1}}^{2}}{100 \mathcal{V}_{\alpha_{1}}^{2} \sum_{n=1}^{N_{d b p}} \log _{10}^{2} d_{n}+\sigma_{1}^{2}} \\
\alpha_{2, o}=20 \frac{\sigma_{2, o}^{2}}{\sigma_{2}^{2}}\left[\alpha 2 \sum_{n=N_{d b p}+1}^{N} y_{n} \log _{10} \frac{d_{n}}{d_{b p}}-\right. \\
\left.-\left(L_{o}+10 \alpha_{1} \log _{10} d_{b p}\right) \sum_{n=N_{d b p}+1}^{N} \log _{10} \frac{d_{n}}{d_{b p}}\right] \\
\sigma_{2,0}^{2}=100 \frac{\mathcal{V}_{\alpha_{2}}^{2} \sum_{n=N_{d b p}+1}^{N} \log _{10} \frac{d_{n}}{d_{b p}}}{\sigma_{2}^{2} \mathcal{V}_{\alpha_{2}^{2}}^{2}}
\end{gathered}
$$

and thus the Bayesian solution based on Gibbs Sampling can be easily implemented where $N$ refers to the total number of samples and $N_{d b p}$ the number of samples before the breakpoint distance. 


\section{Distance Estimation}

Once (1) is calibrated (as in section III), the EKF selects the corresponding model based on the breakpoint distance and the Interactive Multiple Model (IMM) Algorithm developed in [25] is used for distance estimation. Assuming that the mobile target receives RSS measurements from the AP at every $k$ instant, a state vector is defined as $\boldsymbol{\theta}_{k}=\left[\begin{array}{ll}d_{k} & \dot{d}_{k}\end{array}\right]^{T}$ where $d_{k}$ is the distance between the mobile and the AP and $\dot{d}_{k}$ is the rate of change of this distance.

The linear evolution of $\boldsymbol{\theta}_{k}=\mathbf{A} \boldsymbol{\theta}_{k-1}+\mathbf{B} v_{k}$ is assumed where $\mathbf{B} v_{k}$ is the process noise accounting for possible modeling mismatches. The process noise is normally distributed with zero mean and covariance matrix $\mathbf{Q}_{k}=\sigma_{d}^{2} \mathbf{B B}^{T}$ where $\sigma_{d}^{2}$ models the uncertainty on the mobile dynamics. The mobile target has an average velocity of $0.20 \mathrm{~m} / \mathrm{s}$, so a small initial value for $\sigma_{d}^{2}$ of $0.7 \mathrm{~m} / \mathrm{s}^{2}$ is chosen. The state equation includes these matrices:

$$
\mathbf{A}=\left[\begin{array}{cc}
1 & \Delta t \\
0 & 1
\end{array}\right] \quad ; \quad \mathbf{B}=\left[\begin{array}{c}
\frac{\Delta t^{2}}{2} \\
\Delta t
\end{array}\right]
$$

where $\Delta t$ is the sampling period of 0.134 seconds.

The RSS measurements are precisely the observations used to infer $\boldsymbol{\theta}_{k}$, and thus $z_{k} \triangleq \operatorname{RSS}\left(d_{k}\right)=h\left(d_{k}\right)+n_{k}$. Therefore, $h\left(d_{k}\right)$ has to be selected according to (1) and variance of the measurement noise $n_{k}$ is $\hat{\sigma}_{1}^{2}$ or $\hat{\sigma}_{2}^{2}$. The state estimation or filtering is solved using an Extended Kalman Filter (EKF) solution implemented on a IMM algorithm [25] where the corresponding Jacobian matrices $\mathbf{H}_{k}^{(1)}$ and $\mathbf{H}_{k}^{(2)}$ are

$$
\mathbf{H}_{k}^{(1)}=\left[\begin{array}{ll}
\frac{\hat{\alpha}_{1,0}}{\log 10} \frac{10}{d_{k}} & 0
\end{array}\right] ; \mathbf{H}_{k}^{(2)}=\left[\begin{array}{ll}
\frac{\hat{\alpha}_{2}}{\log 10} \frac{10}{d_{k}} & 0
\end{array}\right] .
$$

The initialization for the one-classical model case is, $\mathbf{P}_{0 \mid 0}=$ $4 Q_{k}$. otherwise for two-slope case the error covariance matrix is $\mathbf{P}_{0 \mid 0}=15 \mathbf{Q}_{k}$. The initial value state vector for both filters is $\hat{\boldsymbol{\theta}}_{0 \mid 0}$ which is discussed in Section V-B.

\section{Evaluation}

This section evaluates the proposed algorithms (calibration and distance filtering) with real RSS measurements. The RSS measurements were collected for LOS/NLOS conditions in the same indoor office environment.

\section{A. Experimental setup}

Three experiments were performed:

1) Taking RSS measurements from the same AP in LOS and later in a NLOS condition. The measurements were obtained in a real office environment as shown in Figure 1. The architectural plan is the second floor of a typical multi-story office building with drywall and Wood Wall panelings reinforced with aluminum bars.

2) For accomplishing the NLOS condition, a Wood Wall paneling with a width of 6 centimeters was placed between the AP and the mobile target ( 0.40 meters after the AP shown in figure 1). These RSS measurements

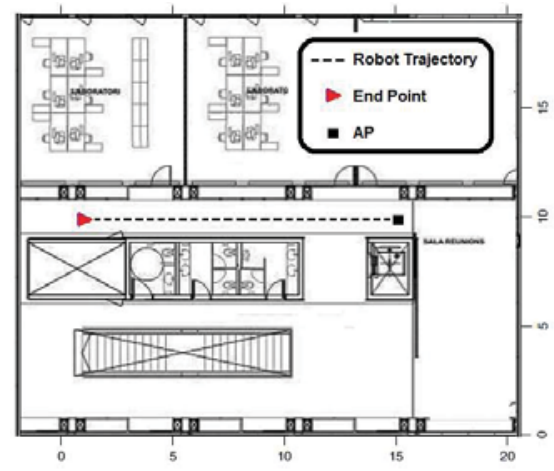

Fig. 1. Office map. Anchor point location and mobile path are marked. The real dmax is 15 meters in straight line between the mobile target and the AP.

were used for the model calibration and distance estimation also.

3) For the tracking task, two algorithms were employed. An EKF-IMM modeled with the classical path loss model and the other with the IMM algorithm developed in [25] for the two-slope model.

The test-bed used to collect the RSS measurements includes a RaspberryP $i$ board. In order to mitigate the antennas orientation problem, the mobile target was always faced up and oriented parallel to the anchor point.

\section{B. Hardware description}

The ranging/positioning payload is a development board with multiple connections where ranging and positioning algorithms can be easily implemented. The RaspberryPi board was the model B with a Universal Serial Bus (USB) WiFi card (IEEE 802.11n, 802.11g, 802.11b). The RaspberryPi board has a Central Processing Unit (CPU) ARM11@700 MHz featuring with a floating point ALU, Ethernet, an Arithmetic Logic Unit (ALU) 2.0, I2C bus, a serial port and Generalpurpose input/outputs (GPIOs), a Linux Operative System (debian based distribution) and a dedicated High-definition camera connector. The positioning payload is a cheap and easy-to-use system that allows WiFi RSS reading with a CPU power (similar to an entry level smartphone).

The overall system consists of the ranging/positioning payload and the database. The RaspberryPi microcontroller sends the RSS measurements to the data base of the server. In the Figure 2, an schematic of the overall system is seen where the ranging/positioning payload reads RSS WiFi measurements employing a TL-WN722N WiFi card (from TP-LINK manufacturer). Data fusion algorithms and ranging models can be implemented and tested in this platform or logged into a database for offline processing purposes.

An integrated navigation information system must continuously know the current position with a good precision thus, a model is needed to measure the real position. The chosen model is a four wheel Robot that is capable of performing a programmed trajectory through waypoints. The main board features an Arduino Platform. Arduino is an open-source 


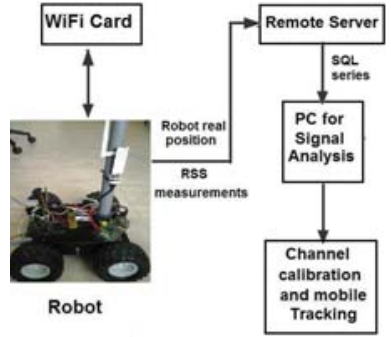

Fig. 2. System connection diagram.

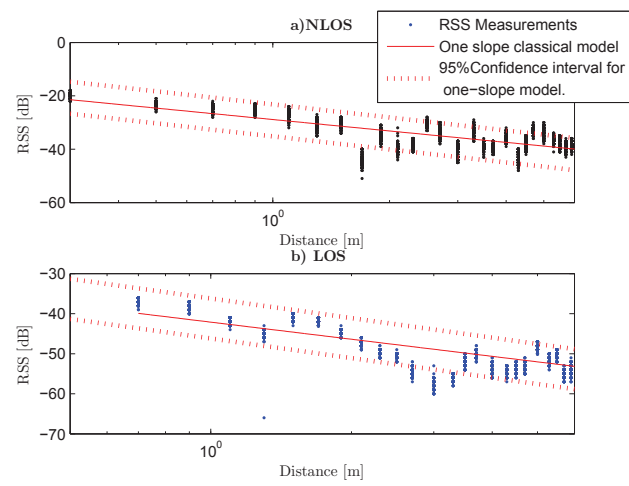

Fig. 3. Classical one-slope channel model.

platform and consists of a physical programmable circuit board (often referred to as a microcontroller) and an IDE (Integrated Development Environment) based in C++ language programming and used for software loading in the board. The initial value for $\hat{\boldsymbol{\theta}}_{0 \mid 0}$ is the first real distance measurement given by the Robot.

\section{RESULTS}

The first step was to obtain a channel model calibration for close distances. Figure 3 shows the RSS measurements taken until 6.7 meters in comparative with the path loss model obtained from our Bayesian inference algorithm proposed for a classical model. From this figure, it can be inferred that for close distances, the classical model is enough for a channel calibration.

The estimated parameter values are detailed in Table I for LOS and NLOS conditions. The difference between estimated channel parameters is due to the indoor conditions described in past sections.

We made a comparative between the classical one-slope model and the two-slope model with the channel model calibration results. The differences between both cases in a LOS condition can be seen in Figure 4. As well, the confidence intervals for the classical model and for the two-slope model are plotted also. From this figure, it is notable that for large distances, the model calibration algorithm for a two-slope model has a best channel parameters estimation in comparative with the classical model.

\begin{tabular}{|c|c|c|c|c|}
\hline \multicolumn{3}{|c|}{ NLOS } & \multicolumn{2}{c|}{ LOS } \\
\hline & Two-Slope & One Slope & Two-Slope & One Slope \\
\hline$\hat{\alpha}_{1}$ & 2.9725 & 1.3245 & 1.9172 & 2.6755 \\
\hline$\hat{\alpha}_{2}$ & 5.4136 & - & 4.8506 & - \\
\hline$\hat{\sigma}_{1}$ & 4.5103 & 3.2406 & 2.3328 & 2.364 \\
\hline$\hat{\sigma}_{2}$ & 6.7040 & - & 4.1013 & - \\
\hline$\hat{d}_{b p}$ & 6.0429 & - & 7.2036 & - \\
\hline
\end{tabular}

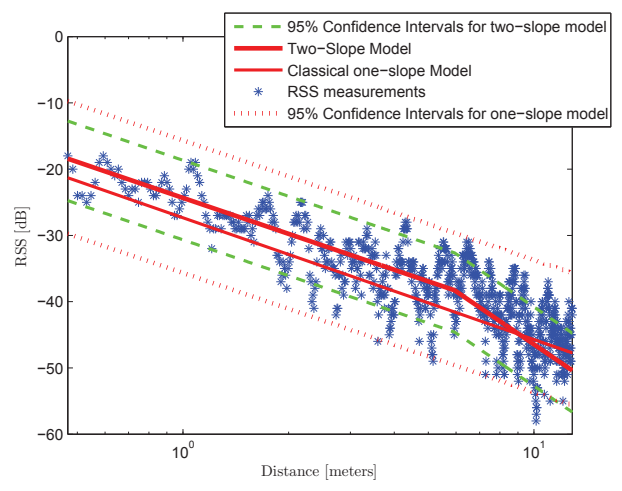

Fig. 4. Two-slope model with real RSS measurements in a LOS scenario.

The marginal distributions for channel parameters and $d_{b p}$ estimation are plotted in Figure 5.

After the channel calibration, the EKF-IMM algorithm was implemented to estimate the distance to the AP in a LOS/NLOS environment. The error between the estimated distance and the real Mobile Target position per every distance interval is shown in the Figure 6. To verify the accuracy of our algorithm, the distance estimation was computed also with an EKF modeled with the classical one-slope model only. From this figure, it can be see the difference in the $d_{b p}$ value for a LOS or NLOS case also. It is notable that for large distances, the EKF-IMM algorithm has a best performance than just considering the classical path loss model only.
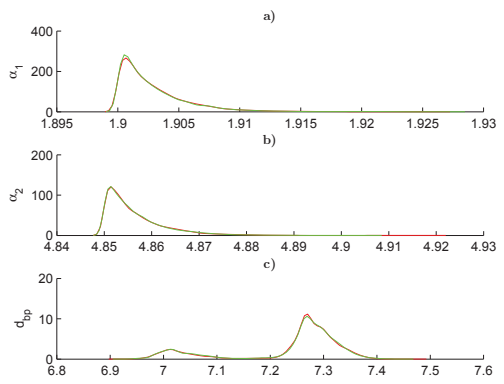

Fig. 5. Marginal Distributions for channel parameters by Bayesian inference for RSS measurementes in a LOS condition. 

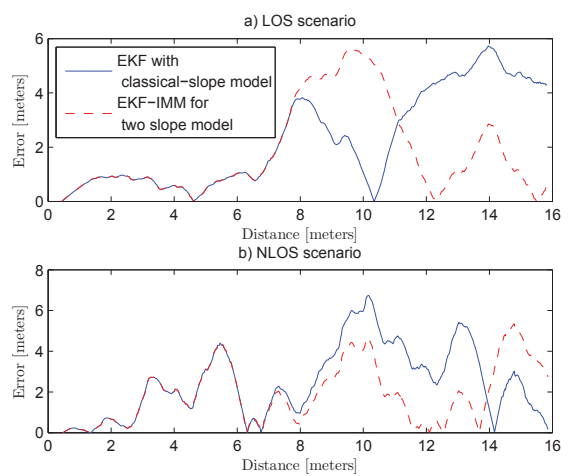

Fig. 6. Error for distance estimation considering a NLOS/LOS scenario.

\section{CONCLUSIONS}

A channel calibration with a two-slope model and distance estimation using real signal was investigated in this work. The calibration method for the two-slope model proposed previously was validated in comparative with the classicalslope model. After model calibration, a distance estimation method was implemented and tested with real data. As future work, developing an online algorithm for NLOS identification and mitigation is a goal as well as mobile tracking in noncontrolled environment as positioning in a real environment where several AP are deployed.

\section{REFERENCES}

[1] Hui Liu, H. Darabi, P. Banerjee, and Jing Liu, "Survey of wireless indoor positioning techniques and systems," Systems, Man, and Cybernetics, Part C: Applications and Reviews, IEEE Transactions on, vol. 37, no. 6, pp. 1067-1080, 2007.

[2] Ahmad Hatami, Application of Channel Modeling for Indoor Localization Using TOA and RSS, PhD dissertation, Worcester Polytechnic Institute, May 2006.

[3] D. Dardari, P. Closas, and P.M. Djuric, "Indoor tracking: Theory, methods, and technologies," Vehicular Technology, IEEE Transactions on, vol. 64, no. 4, pp. 1263-1278, April 2015.

[4] Musa Zeytinci, Veli Sari, Frederic Harmanci, Emin Anarim, and Mehmet Akar, "Location estimation using rss measurements with unknown path loss exponents," EURASIP J. Wireless Comm. and Networking, vol. 2013, pp. 178, 2013.

[5] Xinrong Li, "RSS-Based Location Estimation with Unknown Pathloss Model," Wireless Communications, IEEE Transactions on, vol. 5, no. 12, pp. 3626-3633, December 2006.

[6] K. El-Kafrawy, M. Youssef, A. El-Keyi, and A. Naguib, "Propagation Modeling for Accurate Indoor WLAN RSS-Based Localization," in Vehicular Technology Conference Fall (VTC 2010-Fall), 2010 IEEE 72nd, 2010, pp. 1-5.

[7] K., C. Koweerawong, K. Wipusitwarakun, and Kaemarungsi, "Indoor localization improvement via adaptive rss fingerprinting database," in Information Networking (ICOIN), 2013 International Conference on, Jan 2013, pp. 412-416.

[8] Sinan Gezici, "A Survey on Wireless Position Estimation," Wireless Personal Communications, vol. 44, no. 3, pp. 263-282, February 2008.

[9] David I. Laurenson, Indoor Radio Channel Propagation Modelling by Ray Tracing Techniques, PhD dissertation, University of Edinburgh, 1994.
[10] T. Jamsa, T. Poutanen, and J. Meinila, "Implementation Techniques of Broadband Radio Channel Simulators," in Vehicular Technology Conference, 2001. VTC 2001 Spring. IEEE VTS 53rd, 2001, vol. 1, pp. 433-437 vol.1.

[11] N. Patwari, A.O. Hero, M. Perkins, N.S. Correal, and R.J. O'Dea, "Relative Location Estimation in Wireless Sensor Networks," Signal Processing, IEEE Transactions on, vol. 51, no. 8, pp. 2137-2148, 2003.

[12] M.A. Caceres, F. Sottile, and Spirito, "Adaptive location tracking by Kalman Filter in Wireless Sensor Networks," in Wireless and Mobile Computing, Networking and Communications, 2009. WIMOB 2009. IEEE International Conference on, Oct 2009, pp. 123-128.

[13] T. Roos, P. Myllymaki, and H. Tirri, "A statistical modeling approach to location estimation," Mobile Computing, IEEE Transactions on, vol. 1, no. 1, pp. 59-69, Jan 2002.

[14] S. Mazuelas, A Bahillo, R.M. Lorenzo, P. Fernandez, F.A Lago, E. Garcia, Juan Blas, and E.J. Abril, "Robust indoor positioning provided by real-time rssi values in unmodified wlan networks," Selected Topics in Signal Processing, IEEE Journal of, vol. 3, no. 5, pp. 821-831, Oct 2009.

[15] Hing Cheung So and Lanxin Lin, "Linear least squares approach for accurate received signal strength based source localization," Signal Processing, IEEE Transactions on, vol. 59, no. 8, pp. 4035-4040, Aug 2011.

[16] S. Marano, W.M. Gifford, H. Wymeersch, and M.Z. Win, "NLOS identification and mitigation for localization based on UWB experimental data," Selected Areas in Communications, IEEE Journal on, vol. 28, no. 7, pp. 1026-1035, September 2010

[17] I. Guvenc, Chia-Chin Chong, and F. Watanabe, "NLOS identification and mitigation for UWB localization systems," in Wireless Communications and Networking Conference, 2007.WCNC 2007. IEEE, March 2007, pp. 1571-1576.

[18] Zhuoling Xiao, Hongkai Wen, A. Markham, N. Trigoni, P. Blunsom, and J. Frolik, "Identification and mitigation of non-line-of-sight conditions using received signal strength," in Wireless and Mobile Computing, Networking and Communications (WiMob), 2013 IEEE 9th International Conference on, Oct 2013, pp. 667-674.

[19] T. Paul and T. Ogunfunmi, "Wireless LAN Comes of Age: Understanding the IEEE 802.11n Amendment," Circuits and Systems Magazine, IEEE, vol. 8, no. 1, pp. 28-54, 2008.

[20] J. Manuel Castro-Arvizu, Pau Closas, and J. A. Fernández-Rubio, "Cramér-Rao lower bound for breakpoint distance estimation in a pathloss model," in in Proceedings of IEEE International Conference on Communications (IEEE ICC 2014), 10-14 June 2014,, Sydney (Australia), June 2014.

[21] A.R. Sandeep, Y. Shreyas, Shivam Seth, Rajat Agarwal, and G. Sadashivappa, "Wireless Network Visualization and Indoor Empirical Propagation Model for a Campus Wi-Fi Network," World Academy of Science, Engineering and Technology, vol. 18, no. 6, pp. 705 - 710, 2008.

[22] J.M. Bernardo, Bayesian Statistics, University of Valencia, 46100, Burjassot, Valencia, Spain, November 2001

[23] Andrew Thomas David Spiegelhalter, WinBUGS User Manual, Department of Epidemiology \& Public Health, Imperial College School of Medicine, Norfolk Place, London W2 1PG, UK, 1.4 edition, January 2003

[24] A. F. M. Smith and G. O. Robert, "Bayesian computation via the Gibbs sampler and related Markov Chain Monte Carlo Methods," Journal of the Royal Statistical Society. Series B (Methodological), Vol. 55, No. 1, pp. 3-23, 1993.

[25] J. Manuel Castro-Arvizu, J. Vilà-Valls, Pau Closas, and J. A. FernándezRubio, "Simultaneous tracking and RSS model calibration by robust filtering," in Signals, Systems and Computers, 2014 Asilomar Conference on, Nov 2014. 